\title{
Study of Isolated Contact Temperature Monitoring System Based on Optical Wireless Sensing Technology
}

\author{
Weibing $\mathrm{GAN}^{1}$ and Youfa WANG ${ }^{2}$ \\ ${ }^{1}$ National Engineering Laboratory for Fiber Optic Sensing Technology, Wuhan University of Technology, Wuhan, \\ 430070, China \\ ${ }^{2}$ Hubei Airport Group, Wuhan, 430070, China \\ ${ }^{*}$ Corresponding author: Weibing GAN_ E-mail: ganweibing@whut.edu.cn
}

\begin{abstract}
In view of the high voltage, the strong magnetic field environment of the high-voltage switchgear, the system based on the optical wireless sensing technology, realizes the isolated contact temperature monitoring of the high-voltage switchgear. In this scheme, the good thermal conductivity and insulation ceramic materials were selected as the outer jacket material sensing probe, and the program had a good solution to the problem of high and low voltage isolation; The combination of the optical fiber composite insulators for wireless sensing, not only solves the problem of high voltage insulation, but also avoids the "creeping" phenomenon due to dust. The package of the structure and reasonable installation solves the cross sensitivity of strain. The application showed that the precision of temperature measurement of the system could reach $\pm 0.5^{\circ} \mathrm{C}$ and could work in a variety of harsh environments. It could improve the reliability of the operation of the electrical equipment significantly and has considerable practical value for the whole power system.
\end{abstract}

Keywords: Fiber grating, temperature sensor, composite insulator, high-voltage switchgear, isolated contact

Citation: Weibing GAN and Youfa WANG, "Study of Isolated Contact Temperature Monitoring System Based on Optical Wireless Sensing Technology,” Photonic Sensors, 2014, 4(2): 108-112.

\section{Introduction}

With the rapid development of high voltage and large capacity units of the power system, the reliability of the power supply system has become increasingly demanding, and the safe operation of the electrical equipment is affecting the safe operation of the power system as an important factor. The majority of our high-voltage equipment works in the high voltage, high current, strong magnetic field environment, and the heat is concentrated due to the closed structure, poor cooling conditions, seriously affecting the normal operation of the power equipment [1].

Several common measurement methods are: infrared, wireless radio frequency (RF), surface acoustic wave, and optical fiber thermometry at home and abroad [2-4]. The infrared temperature measurement method cannot accurately measure the contact temperature due to the block of switchgear components; the RF wireless temperature measurement method solves the problem of the measuring equipment and high voltage insulation, but the instability of the power supply and strong electromagnetic interference will cause the instability of the device; the surface acoustic wave

Received: 26 December 2013 / Revised version: 23 February 2014

(C) The Author(s) 2013. This article is published with open access at Springerlink.com

DOI: $10.1007 / \mathrm{s} 13320-014-0161-8$

Article type: Regular 
technology is a good wireless measurement method, but the device is unstable, and the effective transmission distance is short; the distributed optical fiber temperature measurement method is difficult to achieve point positioning measurement, but the optical fiber grating temperature measurement method has the advantage of point measurement and easy installation, and it is especially suitable for cabinet measurement.

In the practical applications of the existing online temperature monitoring technology of the fiber grating in the switchgear, a switchgear contact temperature online monitoring system is included, and this system can overcome the above problems, and can work in a variety of adverse circumstances.

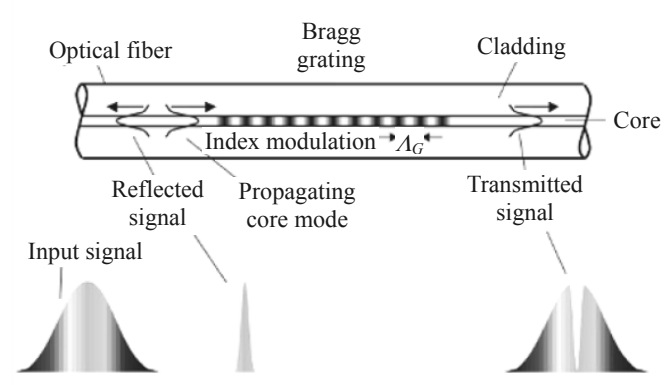

Fig. 1 Diagram of the FBG sensor principle.

Temperature sensing properties of the fiber grating are caused by the thermo-optic effect and thermal expansion effect of the fiber grating, the thermo-optic effect causes the change in the effective refractive index of the fiber Bragg grating rate, and the thermal expansion effect causes a periodic grating grid. When considering the change in the temperature field of the optical fiber grating sensor, we can derive the effect of temperature on the Bragg wavelength changes:

$$
\frac{\Delta \lambda_{B}}{\lambda_{B}}=(\xi+\alpha) \Delta T
$$

where $\alpha$ is the thermal expansion coefficient of the optical fiber, mainly caused by the change in the raster cycle, taking $\alpha$ equal to $5.5 \times 10^{-7}$; $\xi$ is the thermal optical coefficient, mainly caused by the optical fiber refractive index change, taking $\alpha$ equal to $5.5 \times 10^{-6}$.
It can improve the reliability of the operation of the electrical equipment significantly and has considerable practical value for the normal operation of the whole power system.

\section{Principle of fiber grating sensor}

The fiber Bragg grating is an optical device to make a grating structure, and the refractive index of the fiber core has a periodic permanent change by ultraviolet (UV) laser exposure [5]. The optical fiber grating sensor can realize the measurement of temperature and the strain, and the two physical quantities can cause changes in the fiber grating wavelength. The working mechanism is shown in Fig. 1.

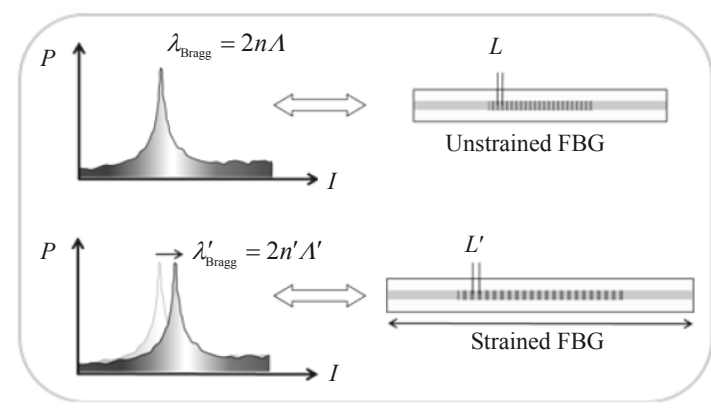

The fiber grating strain sensor is the interaction of the elastic-optic effect and the elastic effect, the raster cycle elastic effects will change the grating period, and the effective index of the elastic-optic effect will change the rate of its sensing characteristics of the fiber. It can be expressed as

$$
\frac{d \lambda_{B}}{\lambda_{B}}=\left(1-P_{e}\right) \varepsilon
$$

where $P_{e}$ is the effective elastic coefficient of the fiber, taking $P_{e}$ equal to 0.22 .

The fiber Bragg grating sensor can measure both the temperature and strain, so we must find a way to shield the effect of strain caused by the switch cabinet vibration.

\section{Design and installation of the sensor}

\subsection{Design of the sensor}

The sensing probe sheath material is a kind of 
heat conduction ceramics, with insulation and high thermal conductivity. The poly tetra fluoro ethylene (PTFE) was selected as the sheath material of the fiber, and it has a low dielectric constant and dielectric over a wide frequency range, the volume resistivity, breakdown voltage, high arc resistance, good insulating performance; at the same time, it also has no oil, no water, corrosion resistance, and other characteristics. It can be very good to avoid flashover due to pollution, wet in the high-voltage environment. The concrete structure of the encapsulated FBG temperature sensor is shown in Fig. 2.

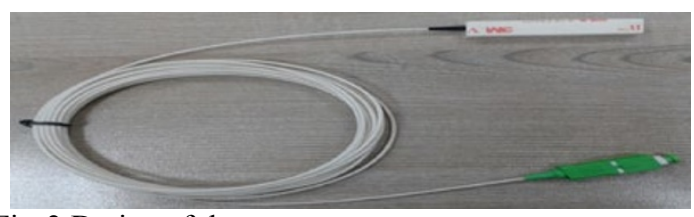

Fig. 2 Design of the sensor.

The size of the packaged sensor was $5 \mathrm{~mm} \times$ $5 \mathrm{~mm} \times 24 \mathrm{~mm}$, the range was from $20{ }^{\circ} \mathrm{C}$ to $220^{\circ} \mathrm{C}$, and it had the advantages of the light weight, convenient installation, and high measuring accuracy. The sensor was placed in a constant temperature water tank after the aging and cyclic test to test the temperature response characteristic, and the temperatures of the thermostat bath were $20{ }^{\circ} \mathrm{C}, 30{ }^{\circ} \mathrm{C}, 40{ }^{\circ} \mathrm{C}, 50{ }^{\circ} \mathrm{C}, 60{ }^{\circ} \mathrm{C}, 70{ }^{\circ} \mathrm{C}, 80{ }^{\circ} \mathrm{C}$, and $90{ }^{\circ} \mathrm{C}$. We used the least-squares linear fit of the test data and found the relationship between the reflection wavelength and temperature, as shown in Fig. 3.

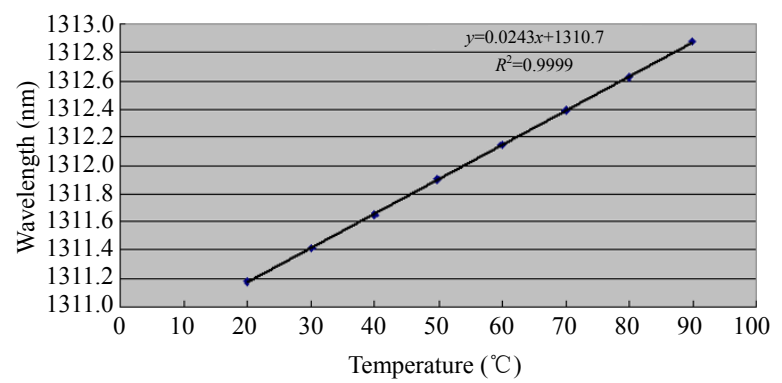

Fig. 3 Analysis of the sensor linearity.

The linear fitting formula is

$$
y=0.0243 x+1310.700 .
$$

The fitting degree was up to 0.9999 , and it can be seen that the changes in the central wavelength had a good linear relation with the temperature.

\subsection{Installation of the sensor}

Six contacts of the high-voltage switchgear were distributed in the upper and lower A, B, C three-phase, so in order to ensure the reliability of the system, six contacts must be monitored simultaneously. The fiber grating sensor is sensitive to temperature and strain, and we must shield the sensitivity of the strain effect, namely any strain of circuit breakers generated in the process should not be passed to the sensor. The sensor was fixed in the static contact in order to shield strain produced in the collision process. In addition, in order to guarantee the uniformity of the sensors on the contact points of the temperature measurement system, the system made full use of the intermediate space of static contact, and the sensors were fixed at the middle position of the stationary contact. Figure 4 is the schematic diagram of the sensor in a single static contact installed.

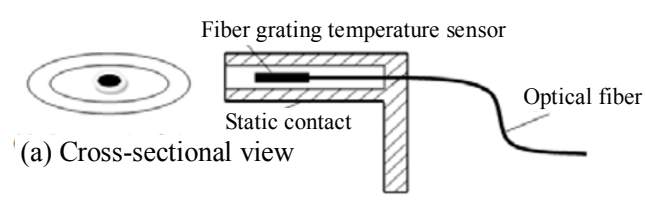

(b) A cross-sectional view

Fig. 4 Installation on the static contact.

When the contact is operated, the sides of static contact will produce strain, and in the center, there is no strain, so the strain cannot be passed to the sensor, which not only guarantees the temperature measurement accuracy but also is blocked due to the vibration caused by the cross sensitivity of the strain.

\section{Optical wireless sensing method}

\subsection{Wireless sensing device}

The fiber composite insulator is the main artifact of wireless sensing, and a pair of optical fiber collimators were embedded on the ends in the middle of them. The collimator consisted of the 
single-mode pigtailed and collimating lens (GRIN lens or C-lens), and it has the advantages of the low insertion loss, high return loss, small volume, light weight, and other characteristics. It can be emergent divergent beam transformation for the parallel beam, or the parallel beam convergence and high efficiency coupled into the optical fiber. In order to solve the "creeping" phenomenon caused by the wet environment and pollution, the device chose a resin material for filling and packaging the composite insulator mandrel, which had the good performance stability, impact toughness, and acid corrosion. The encapsulation principle is shown in Fig. 5.

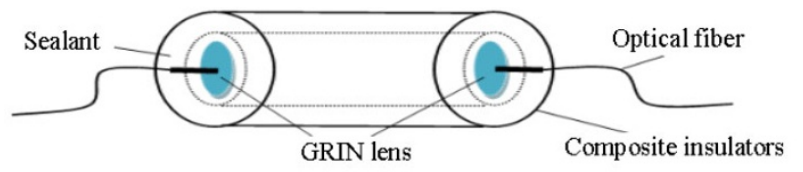

Fig. 5 Diagram of the optical wireless sensing device.

\subsection{Optical fiber network}

Sensors for simultaneous measurement involve the multiplexing optical path, and multiplexed fiber grating sensors can be used in wavelength division multiplexing (WDM), space division multiplexing (SDM) or time division multiplexing (TDM) modes. As shown in Fig. 6, this system adopted the methods of SDM and WDM. SDM can avoid the disadvantages of the single wavelength division multiplexing, and multiple sensors were connected in series in a single optical fiber transmission.

Furthermore, the wavelength division multiplexing method was used to improve the measuring speed of the system in the wavelength selection and division multiplexing, and a plurality of measuring sensors can be achieved simultaneously through a scanning cycle by the WDM technology.

We can see that the six sensors from the A, B, C three-phase in the high voltage side were separately arranged on the static contact aperture, as shown in Fig. 6. Each sensor independently accessed the optical wireless sensing device, and this non-connection mode in series can effectively avoid "creeping" phenomenon of the switch cabinet cable among three electrodes.

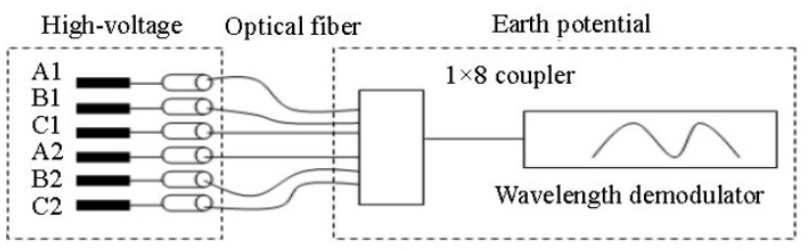

Fig. 6 Optical multiplexing scheme of the system.

\section{Application in the switchgear}

The $10-\mathrm{kV}$ high voltage switchgears were used for temperature monitoring in substation, and six optical fiber temperature sensors were connected in a star connection way. The installation and wiring diagrams of the sensors are shown in Fig. 7.

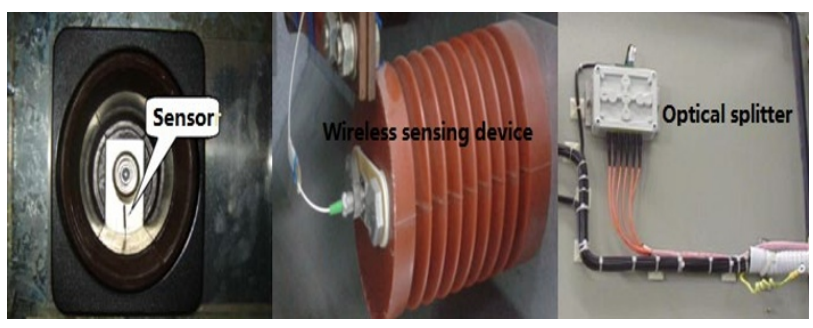

Fig. 7 Installation and wiring diagram.

The optical wireless sensing device was installed in the cabinet with the bolt fixed on the side of a cabinet, and the optical signal from a high voltage side was transmitted to the low voltage side by the optical fiber collimator, so this way solves the problem of high voltage insulation.

As shown in Fig. 8, the data were collected in the actual operation. In order to determine the actual effect of the optical fiber grating temperature measurement system, this experiment was mainly carried out on the basis of the analysis from the following aspects: the measured maximum temperature, minimum temperature, and integrity of the data.

We can see that site equipment operation is normal, and the data are relatively stable. 


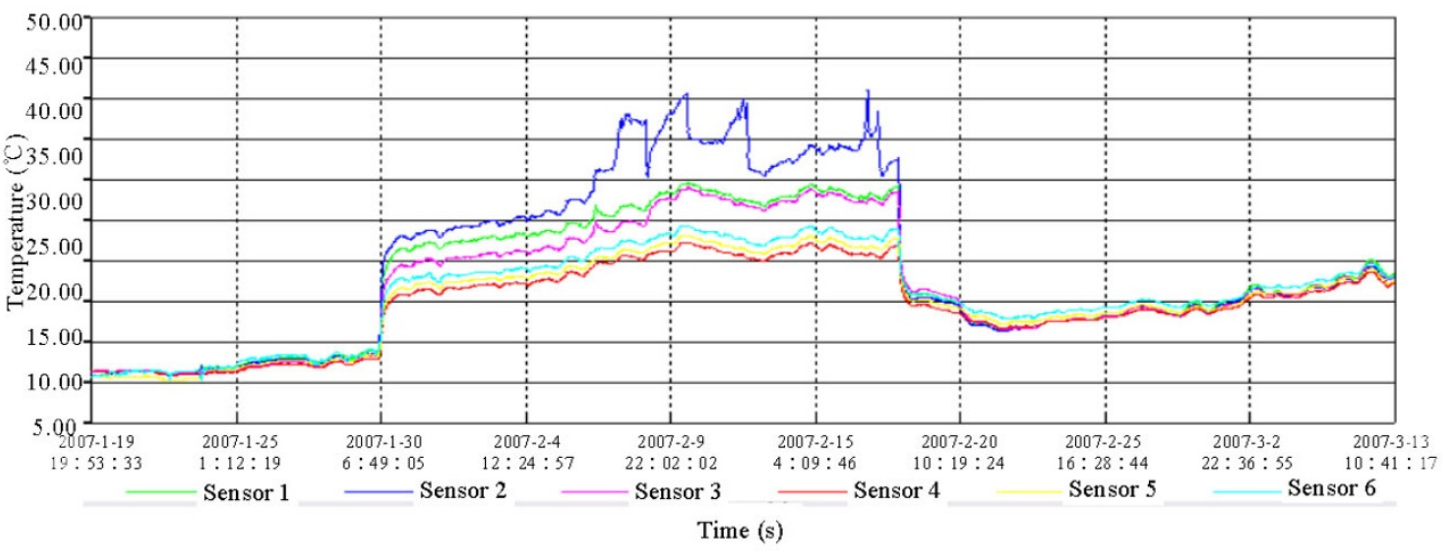

Fig. 8 Curve of temperature measurement.

\section{Conclusions}

In this paper, Fiber Bragg grating sensors were used to achieve the temperature monitoring of the isolated contacts in the high voltage switchgear instead of the electronic sensor, and this scheme did not require the complex insulation design, had the advantages of simple operation and reliability. In this scheme, the system can solve the cross sensitivity through the clever design of the sensor and reasonable installation, and the measuring accuracy was $\pm 0.5^{\circ} \mathrm{C}$. This system experienced a long time test in the $10-\mathrm{kV}$ high voltage switchgear, and the developed sensor had the high stability, high reliability, high performance-price ratio etc. The optical fiber sensor temperature monitoring system is well worth spreading.

Open Access This article is distributed under the terms of the Creative Commons Attribution License which permits any use, distribution, and reproduction in any medium, provided the original author(s) and source are credited.

\section{References}

[1] B. Shi, "HV switchgear temperature on-line monitoring system with distributed optical fiber sensor," High Voltage Engineering, 2007, 33(8): 169-173.

[2] B. Wang and J. Li, "Real-time temperature measurement system of high voltage switchgear by RF wireless technical," Zhejiang Electric Power, 2009, 3: 31-33.

[3] M. Xu and X. YANG, "Wireless monitoring system of temperature for the high voltage switch contact," Measurement Control Technology and Instruments, 2009, 4: 98-101.

[4] X. Qian and X. Wang, "FBG based sensing system for temperature monitoring of the high voltage apparatus," Proc. of the CSU-EPSA, 2007, 19(5): $49-51$.

[5] X. Sun, "Design of switchgear temperature on-line monitoring system based on optical fiber grating," Telecommunications for Electric Power System, 2012, 33(235): 6-10. 